Pacific Journal of Mathematics

WEYL'S INEQUALITY AND QUADRATIC FORMS ON TH 


\title{
WEYL'S INEQUALITY AND QUADRATIC FORMS ON THE GRASSMANNIAN
}

\author{
Patricia Andresen and Marvin Marcus
}

This paper is concerned with the largest absolute value taken on by an $m$-square principal subdeterminant in any unitary transform of an $n$-square complex matrix $A$. For $m=1$ this maximum coincides with the numerical radius of $A$. The results obtained constitute generalizations of the Gohberg-Krein analysis of the case of equality in Weyl's inequalities relating eigenvalues and singular values.

Introduction. Let $A$ be an $n$-square complex matrix with eigenvalues $\lambda_{1}, \cdots, \lambda_{n},\left|\lambda_{1}\right| \geqq \cdots \geqq\left|\lambda_{n}\right|$, and singular values $\alpha_{1}(A) \geqq \cdots \geqq$ $\alpha_{n}(A)$. The numerical radius of $A, r(A)$, is the maximum absolute value assumed by a diagonal element in any unitary transform of of $A$, i.e., in any matrix unitarily similar to $A$. Of course,

$$
\left|\lambda_{1}\right| \leqq r(A)
$$

Matrices for which equality holds in (1) are called spectral. In this paper we consider $r_{d, m}(A)$, the largest absolute value taken on by an $m$-square principal subdeterminant in any unitary transform of $A$. As we shall see in the sequel

$$
\left|\lambda_{1} \cdots \lambda_{m}\right| \leqq r_{d, m}(A) \text {. }
$$

For $m=1$, (2) collapses to (1). Matrices for which equality holds in (2) will be called m-decomposably spectral. One of the purposes of this paper is to examine the structure of matrices $A$ which are $m$-decomposably spectral for each $m=1, \cdots, n$. Such results are related to the inequalities of Weyl [5],

$$
\left|\lambda_{1} \cdots \lambda_{k}\right| \leqq \alpha_{1}(A) \cdots \alpha_{k}(A), k=1, \cdots, n,
$$

and to the case of equality in (3) for $k=1, \cdots, n$ discussed by Gohberg and Krein [1]. We also examine the case where $A$ is $m$-decomposably spectral for a particular $m$ and, in fact, show that if $A$ has $s$ eigenvalues of maximum modulus $\left|\lambda_{1}\right|, s>m$, then spectral and $m$-decomposably spectral are equivalent. To examine the concept of $m$-decomposably spectral we require the machinery of induced maps on the $m$ th Grassmann space.

2. Preliminary notions and theorems. Let $V$ be an $n$-dimensional unitary space with an inner product $(x, y)$. Let $T: V \rightarrow V$ be 
a linear transformation with eigenvalues $\lambda_{1}, \cdots, \lambda_{n},\left|\lambda_{1}\right| \geqq \cdots \geqq\left|\lambda_{n}\right|$, and singular values $\alpha_{1}(T) \geqq \cdots \geqq \alpha_{n}(T)$. Let $E=\left\{e_{1}, \cdots, e_{n}\right\}$ be an o.n. basis of $V$ and let $A=[T]_{E}^{E}$, the matrix representation of $T$ with respect to $E$. We will consider $A$ as a linear transformation on $C^{n}$, the space of complex $n$-tuples. For each $m, 1 \leqq m \leqq n$, let $\Lambda^{m} V$ be the $m$ th Grassmann space over $V$ where the inner product induced on $\Lambda^{m} V$ by $(x, y)$ is defined by

$$
\left(x_{1} \wedge \cdots \wedge x_{m}, y_{1} \wedge \cdots \wedge y_{m}\right)=\operatorname{det}\left[\left(x_{i}, y_{j}\right)\right]
$$

for any decomposable tensors $x^{\wedge}$ and $y^{\wedge}$ in $\Lambda^{m} V$, i.e., $x^{\wedge}=x_{1} \wedge \cdots \wedge x_{m}$, $y^{\wedge}=y_{1} \wedge \cdots \wedge y_{m}$ where $x_{i}$ and $y_{i}$ are in $V, i=1, \cdots, m$. The space $\Lambda^{m} V$ has an ordered o.n. basis $E^{\wedge}=\left\{e_{\omega(1)} \wedge \cdots \wedge e_{\omega(m)}=e_{\omega}^{\wedge}: \omega \in Q_{m, n}\right\}$ where $Q_{m, n}$ is the totality of strictly increasing sequences $\omega$ of length $m, 1 \leqq \omega(1)<\cdots<\omega(m) \leqq n$, and where the $\omega$ 's are assumed to be ordered lexicographically. The compound $C_{m}(T): \bigwedge^{m} V \rightarrow \Lambda^{m} V$ is defined by

$$
C_{m}(T) x_{1} \wedge \cdots \wedge x_{m}=T x_{1} \wedge \cdots \wedge T x_{m}
$$

for any decomposable $x^{\wedge} \in \bigwedge^{m} V$. Let $C_{m}(A)=\left[C_{m}(T)\right]_{E}^{E \wedge}$. Then $C_{m}(A)$ has eigenvalues $\lambda_{\beta}=\lambda_{\beta(1)} \cdots \lambda_{\beta(m)}, \beta \in Q_{m, n}$ and singular values $\alpha_{r}=$ $\alpha_{r(1)}(A) \cdots \alpha_{r(m)}(A), \gamma \in Q_{m, n}$.

The numerical radius of $A$ is defined by

$$
r(A)=\max _{\|x\|=1}|(A x, x)| .
$$

and the spectral norm of $A$ by

$$
\alpha_{1}(A)=\max _{\|x\|=1}\|A x\| \text {. }
$$

The Grassmannian in $\bigwedge^{m} V$ is the set

$$
G_{m}=\left\{x^{\wedge} \in \Lambda^{m} V:\left\|x^{\wedge}\right\|=1 \text { and } x^{\wedge} \text { is decomposable }\right\},
$$

and the decomposable numerical radius of $C_{m}(A)$ is defined by

$$
r_{d}\left(C_{m}(A)\right)=\max _{x^{\wedge} \in G_{m}}\left|\left(C_{m}(A) x^{\wedge}, x^{\wedge}\right)\right| \text {. }
$$

In (4) we may assume without loss of generality that for each $x^{\wedge}=$ $x_{1} \wedge \cdots \wedge x_{m}$ the vectors $x_{1}, \cdots, x_{m}$ are o.n. Since the $\alpha, \beta$ entry of $C_{m}(A)$ is $\operatorname{det} A[\alpha \mid \beta]$, where $A[\alpha \mid \beta]$ indicates the submatrix of $A$ lying in rows $\alpha$ and columns $\beta, \alpha, \beta \in Q_{m, n}$, we see that by taking $U e_{i}=x_{i}, i=1, \cdots, m, U$ unitary, we have

$$
\begin{aligned}
r_{d}\left(C_{m}(A)\right) & =\max _{x^{\wedge} \in G_{m}}\left|\left(C_{m}(A) x^{\wedge}, x^{\wedge}\right)\right| \\
& =\max _{U \text { unitary }}\left|\left(C_{m}(A) C_{m}(U) e_{1} \wedge \cdots e_{m}, C_{m}(U) e_{1} \wedge \cdots \wedge e_{m}\right)\right|
\end{aligned}
$$


WEYL'S INEQUALITY AND QUADRATIC FORMS ON THE GRASSMANNIAN 279

$$
\begin{aligned}
& =\max _{U \text { unitary }}\left|\operatorname{det} U^{*} A U[1, \cdots, m \mid 1, \cdots, m]\right| \\
& =r_{d, m}(A)
\end{aligned}
$$

Of course if $m=1, r_{d}\left(C_{m}(A)\right)=r(A)$. In general,

$$
r_{d}\left(C_{m}(A)\right) \leqq r\left(C_{m}(A)\right)
$$

It is possible to have strict inequality in (5) as the following example shows. Let

$$
A=\left[\begin{array}{llll}
0 & 0 & 1 & 0 \\
0 & 0 & 0 & 1 \\
0 & 0 & 0 & 0 \\
0 & 0 & 0 & 0
\end{array}\right]
$$

so that

$$
C_{2}(A)_{\alpha, \beta}=\left\{\begin{array}{l}
1, \text { if } \alpha=(12), \beta=(34) \\
0, \text { otherwise } .
\end{array}\right.
$$

If $x^{\wedge} \in G_{m}$ then $x^{\wedge}=\sum_{\alpha \in Q_{2,4}} p(\alpha) e_{\alpha}^{\wedge}$ where

(6)

$$
\sum_{\alpha \in Q_{2,4}}|p(\alpha)|^{2}=1
$$

and the $p(\alpha)$ satisfy the quadratic Plücker relations [4]:

$$
p(\alpha) p(\beta)=\sum_{t=1}^{m} p(\alpha[s, t: \beta]) p(\beta[t, s: \alpha], s=1, \cdots, m
$$

where $\alpha[s, t: \beta]$ is the sequence $(\alpha(1), \cdots, \alpha(s-1), \beta(t), \alpha(s+1), \cdots$, $\alpha(m)$ ) and $p(\alpha)$ is defined for any sequence $\alpha$ of length $m$ by skewsymmetry. We have for $x^{\wedge} \in G_{m}$

(8)

$$
\begin{aligned}
\left|\left(C_{2}(A) x^{\wedge}, x^{\wedge}\right)\right| & =|p(12) p(34)| \\
& =|p(32) p(14)+p(42) p(31)|, \quad(\text { from (7) with } s=1) \\
& \leqq|p(23)||p(14)|+|p(24)||p(13)| \\
& \leqq \frac{1}{2}\left(|p(23)|^{2}+|p(14)|^{2}+|p(24)|^{2}+|p(13)|^{2}\right) \\
& =\frac{1-|p(12)|^{2}-|p(34)|^{2}}{2}, \quad(\text { from }(6)) .
\end{aligned}
$$

Thus

$$
\begin{aligned}
& (|p(12)|+|p(34)|)^{2} \leqq 1 \\
& (|p(12)|+|p(34)|)=c \leqq 1 \\
& |p(12)||p(34)|=|p(12)|(c-|p(12)|),
\end{aligned}
$$


and

$$
\mid\left(p(12) p(34) \mid \leqq \frac{c^{2}}{4} \leqq \frac{1}{4} .\right.
$$

From (8) we see that

$$
r_{d}\left(C_{2}(A)\right) \leqq \frac{1}{4}
$$

If we consider the quadratic form evaluated on the indecomposable unit tensor $1 / \sqrt{2}\left(e_{1} \wedge e_{2}+e_{3} \wedge e_{4}\right)$ we have

$$
\left(C_{2}(A) \frac{1}{\sqrt{2}}\left(e_{1} \wedge e_{2}+e_{3} \wedge e_{4}\right), \frac{1}{\sqrt{2}}\left(e_{1} \wedge e_{2}+e_{3} \wedge e_{4}\right)=\frac{1}{2},\right.
$$

so that

$$
r\left(C_{2}(A)\right) \geqq \frac{1}{2}
$$

The explanation of this phenomenon is that not every tensor on the unit sphere in $\Lambda^{2} V$ is decomposable.

The following results are well known [3]:

(i) For $M$ any principal sub-matrix of $A$,

$$
r(M) \leqq r(A) .
$$

(ii) (The Elliptical Range Theorem.) For a $2 \times 2$ matrix the numerical range is an ellipse with foci the eigenvalues of the matrix; if $A=\left[\begin{array}{cc}\lambda_{1} & a \\ 0 & \lambda_{2}\end{array}\right]$ then the semi-minor axis of the ellipse has length $|a| / 2$.

$$
\left|\lambda_{1}\right| \leqq r(A) \leqq \alpha_{1}(A)
$$

We may generalize (10) for $1 \leqq m \leqq n$ to

$$
\left.\left|\lambda_{1} \cdots \lambda_{m}\right| \leqq r_{d}\left(C_{m}(A)\right) \leqq r\left(C_{m} A\right)\right) \leqq \alpha_{1}(A) \cdots \alpha_{m}(A) .
$$

The first inequality may be seen as follows. Let

$$
U^{*} A U=\left[\begin{array}{lll}
\lambda_{1} & & * \\
& \ddots & \\
& & \lambda_{n}
\end{array}\right] .
$$

Then $C_{m}\left(U^{*} A U\right)$ is also upper triangular and

$$
\lambda_{1} \cdots \lambda_{m}=C_{m}\left(U^{*} A U\right)_{(1 \cdots, m),(1, \cdots, m)}=\left(C_{m}(A) u^{\wedge}, u^{\wedge}\right)
$$

for an appropriate $u^{\wedge} \in G_{m}$. If $A$ is normal then equality holds throughout (10) and (11). A proof of the Weyl inequalities (3) is 
now immediate. The first follows from (10) and the subsequent ones from (11). Since $r_{d, m}(A)=r_{d}\left(C_{m}(A)\right)$ we will say that $C_{m}(A)$, $1 \leqq m \leqq n$, is decomposably spectral if

$$
\left|\lambda_{1} \cdots \lambda_{m}\right|=r_{d}\left(C_{m}(A)\right) \text {. }
$$

M. Goldberg, E. Tadmor and G. Zwas [2] have shown that if $\left|\lambda_{1}\right|=$ $\cdots=\left|\lambda_{s}\right|>\left|\lambda_{s+1}\right| \geqq \cdots \geqq\left|\lambda_{n}\right|$ then $A$ is spectral iff $A$ is unitarily similar to a matrix of the form $T+B$ where

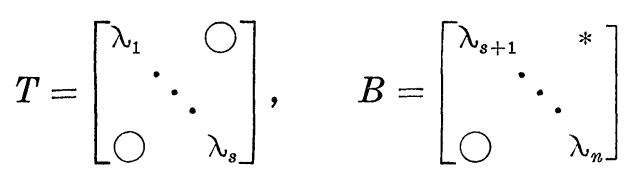

and

$$
r(B) \leqq\left|\lambda_{1}\right| \text {. }
$$

Theorem 1 (Gohberg and Krě̃n). Equality holds in (3) for $k=1, \cdots, n$ iff $A$ is normal.

We include a proof of this theorem based on properties of the Grassmann algebra which suggests a proof of the following stronger result:

Theorem 2. For each $m=1, \cdots, n$

$$
\left|\lambda_{1} \cdots \lambda_{m}\right| \leqq r_{d}\left(C_{m}(A)\right), \quad m=1, \cdots, n .
$$

Equality holds in (13) for $m=1, \cdots, n$ iff $A$ is normal. Equivalently, the largest absolute value taken on by an m-square principal subdeterminant in any unitary transform of $A$ is at least $\left|\lambda_{1} \cdots \lambda_{m}\right|$, $m=1, \cdots, n$. This largest absolute value is equal to $\left|\lambda_{1} \cdots \lambda_{m}\right|$ for $m=1, \cdots, n$ iff $A$ is normal.

We will also investigate the case of equality in a single one of the inequalities in (13).

Theorem 3. Assume that $A$ has $s$ eigenvalues of maximum modulus, $s>m$ :

$$
\left|\lambda_{1}\right|=\cdots=\left|\lambda_{s}\right|>\left|\lambda_{s+1}\right| \geqq \cdots \geqq\left|\lambda_{n}\right| \cdot
$$

Then $C_{m}(A)$ is decomposably spectral iff $A$ is spectral.

3. Proofs and examples.

Proof of Theorem 1. Clearly if $A$ is normal then $\left|\lambda_{1} \cdots \lambda_{k}\right|=$ 
$\alpha_{1}(A) \cdots \alpha_{k}(A), k=1, \cdots, n$. Suppose now that $\left|\lambda_{1} \cdots \lambda_{k}\right|=\alpha_{1}(A)_{j}^{3} \cdots$ $\alpha_{k}(A), k=1, \cdots, n$. By Schur's theorem we may assume

$$
A=\left[\begin{array}{lll}
\lambda_{1} & & * \\
& \ddots & \\
& \ddots & \lambda_{n}
\end{array}\right]
$$

Let

$$
\left|\lambda_{1}\right| \geqq \cdots \geqq\left|\lambda_{t}\right|>0=\left|\lambda_{t+1}\right|=\cdots=\left|\lambda_{n}\right|,
$$

for some $t, 1 \leqq t \leqq n$. We have

$$
\begin{aligned}
\left(A A^{*}\right)_{11} & =\left|\lambda_{1}\right|^{2}+\left|a_{12}\right|^{2}+\cdots+\left|\alpha_{1 n}\right|^{2} \\
& \leqq \alpha_{1}^{2}(A) .
\end{aligned}
$$

Since $\left|\lambda_{1}\right|=\alpha_{1}(A)$ we must have $\alpha_{l i}=0, i \neq 1$ and

$$
A_{(1)}=\lambda_{1} e_{1} \text {. }
$$

( $A_{(1)}$ is the first row of $A$, i.e., the $n$-tuple $\left(a_{11}, \cdots, a_{1 n}\right)$.) Applying this argument to $C_{m}(A), 1 \leqq m \leqq n$, we have

$$
\begin{aligned}
C_{m}(A)_{(1)} & =A_{(1)} \wedge \cdots \wedge A_{(m)} \\
& =\lambda_{1} \cdots \lambda_{m} e_{1} \wedge \cdots \wedge e_{m} .
\end{aligned}
$$

Assume now that we have shown

$$
A_{(i)}=\lambda_{i} e_{i}, i=1, \cdots, k-1, k \leqq t .
$$

Then

$$
\begin{aligned}
A_{(1)} & \wedge \cdots \wedge A_{(k)}=\lambda_{1} \cdots \lambda_{k-1} e_{1} \wedge \cdots \wedge e_{k-1} \wedge\left(\lambda_{k} e_{k}+\sum_{i=k+1}^{n} a_{k i} e_{i}\right) \\
& =\lambda_{1} \cdots \lambda_{k} e_{1} \wedge \cdots \wedge e_{k}+\lambda_{1} \cdots \lambda_{k-1}\left(\sum_{i=k+1}^{n} a_{k i} e_{1} \wedge \cdots \wedge e_{k-1} \wedge e_{i}\right) .
\end{aligned}
$$

Since the representation of $A_{(1)} \wedge \cdots \wedge A_{(k)}$ with respect to the basis $E^{\wedge}$ is unique and since $\lambda_{1} \cdots \lambda_{k} \neq 0,(14)$ and (16) imply $a_{k i}=0, i=$ $k+1, \cdots, n$. We have

$$
A=\operatorname{diag}\left(\lambda_{1}, \cdots, \lambda_{t}\right)+B
$$

where

$$
B=\left[\begin{array}{lll}
0 & & * \\
& \ddots & \\
& & \\
0 & & 0
\end{array}\right] \text {. }
$$

However, $\left|\lambda_{1} \cdots \lambda_{t+1}\right|=\alpha_{1}(A) \cdots \alpha_{t+1}(A)$ implies that 


$$
\alpha_{t+1}(A)=\cdots=\alpha_{n}(A)=0 .
$$

Thus $A A^{*}$, and hence $A$, has rank $t$ so that $B=0_{n-t}$. Thus $A$ is normal.

Proof of Theorem 2. If $A$ is normal then obviously equality holds in (13) for $m=1, \cdots, n$. Conversely, assume that (13) is equality, $m=1, \cdots, n$. Without loss of generality we can assume

$$
A=\left[\begin{array}{lll}
\lambda_{1} & & * \\
& \ddots & \\
& & \lambda_{n}
\end{array}\right] .
$$

Suppose there exists an $a_{1 i}, i \neq 1$, such that $a_{12}$ is nonzero. Then from (9),

$$
\left|\lambda_{1}\right|=r(A) \geqq r\left[\begin{array}{cc}
\lambda_{1} & a_{1 i} \\
0 & \lambda_{i}
\end{array}\right] \geqq\left|\lambda_{1}\right|,
$$

so that by the Elliptical Range Theorem $a_{1 i}=0$ and

$$
A_{(1)}=\lambda_{1} e_{1} \text {. }
$$

Let

$$
\left|\lambda_{1}\right| \geqq \cdots \geqq\left|\lambda_{t}\right|>0=\left|\lambda_{t+1}\right|=\cdots=\left|\lambda_{n}\right|
$$

for some $t, 1 \leqq t \leqq n$, and suppose we have shown that

$$
A_{(i)}=\lambda_{i} e_{i}, i=1, \cdots, k-1, k \leqq t .
$$

Let $1 \leqq r \leqq n-k$ and consider the function

$$
\begin{aligned}
& e(u, v)=\left(C_{k}(A) e_{1} \wedge \cdots \wedge e_{k-1} \wedge\left(u e_{k}+v e_{k+r}\right),\right. \\
&\left.e_{1} \wedge \cdots \wedge e_{k-1} \wedge\left(u e_{k}+v e_{k+r}\right)\right)
\end{aligned}
$$

where $|u|^{2}+|v|^{2}=1$. Then

$e(u, v)$

$$
\begin{aligned}
= & \left(\lambda_{1} \cdots \lambda_{k-1}\left(u \lambda_{k} e_{1} \wedge \cdots \wedge e_{k-1} \wedge e_{k}+v \sum_{i=k}^{k+r} \alpha_{i, k+r} e_{1} \wedge \cdots \wedge e_{k-1} \wedge e_{i}\right),\right. \\
& \left.u e_{1} \wedge \cdots \wedge e_{k-1} \wedge e_{k}+v e_{1} \wedge \cdots \wedge e_{k-1} \wedge e_{k+r}\right) \\
= & \lambda_{1} \cdots \lambda_{k-1}\left\{|u|^{2} \lambda_{k}+v \bar{u} a_{k, k+r}+|v|^{2} \lambda_{k+r}\right\}
\end{aligned}
$$

Let

$$
C=\left[\begin{array}{cc}
\lambda_{k} & a_{k, k+r} \\
0 & \lambda_{k+r}
\end{array}\right]
$$


If $a_{k, k+r} \neq 0$ then from the Elliptical Range Theorem $r(C)>\left|\lambda_{k}\right|$, i.e., there exist $u$ and $v,|u|^{2}+|v|^{2}=1$, such that the expression in curly brackets on the right side of (17) has absolute value greater than $\left|\lambda_{k}\right|$. Since $\lambda_{1} \cdots \lambda_{k-1}$ is nonzero we conclude that $|e(u, v)|>\left|\lambda_{1} \cdots \lambda_{k}\right|$. But $e(u, v)$ is a value of the quadratic form associated with $C_{k}(A)$ on a decomposable tensor of unit length, and thus it follows that $r_{d}\left(C_{k}(A)\right)>\left|\lambda_{1} \cdots \lambda_{k}\right|$. Therefore $a_{k, k+r}=0, r=1, \cdots, n-k$ and thus

$$
A=\operatorname{diag}\left(\lambda_{1} \cdots \lambda_{t}\right)+B
$$

where

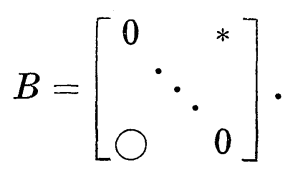

Next assume $a_{t+1, i} \neq 0$ for some $i>t+1$. Then the $(1, \cdots, t, t+1)$, $(1, \cdots, t, i)$ element of $C_{t+1}(A)$ is $\lambda_{1} \cdots \lambda_{t} a_{t+1, i} \neq 0$. Letting $x^{\wedge}$ be the decomposable unit tensor $1 \sqrt{2}\left(e_{1} \wedge \cdots \wedge e_{t} \wedge e_{t+1}+e_{1} \wedge \cdots \wedge e_{t} \wedge e_{i}\right)$ we have

$$
\begin{aligned}
\left(C_{t+1}(A) x^{\wedge}, x^{\wedge}\right)= & \frac{1}{2}\left(\lambda_{1} \cdots \lambda_{t} e_{1} \wedge \cdots \wedge e_{t} \wedge\left(a_{t+1, i} e_{t+1}+\sum_{j=t+2}^{n} a_{j \imath} e_{j}\right),\right. \\
& \left.e_{1} \wedge \cdots \wedge e_{t} \wedge e_{t+1}+e_{1} \wedge \cdots \wedge e_{t} \wedge e_{i}\right) \\
= & \frac{1}{2} \lambda_{1} \cdots \lambda_{t} a_{t+1, i} \\
& \neq 0 .
\end{aligned}
$$

But then $r_{d}\left(C_{t+1}(A)\right) \geqq 1 / 2\left|\lambda_{1} \cdots \lambda_{t} a_{t+1, i}\right|>\left|\lambda_{1} \cdots \lambda_{t} \lambda_{t+1}\right|=0$, contradicting the assumption that (13) is equality for $m=t+1$. Thus

$$
A_{(t+1)}=0 \text {. }
$$

Suppose that we have shown

$$
A_{(t+r)}=0, r=1, \cdots, k-1 .
$$

If there exists an element $a_{t+k, i}, i>t+k$, which is nonzero we see that the $(1, \cdots, t, t+k),(1, \cdots, t, i)$ element of $C_{t+1}(A)$ is $\lambda_{1} \cdots \lambda_{t} a_{t+k, i} \neq 0$. Let $x^{\wedge}=1 / \sqrt{2}\left(e_{1} \wedge \cdots \wedge e_{t} \wedge e_{t+k}+e_{1} \wedge \cdots \wedge e_{t} \wedge e_{i}\right) \in G_{t+1}$ and note that

$$
\begin{aligned}
\left(C_{t+1}(A) x^{\wedge}, x^{\wedge}\right) & =\frac{1}{2} \lambda_{1} \cdots \lambda_{t} a_{t+k, i} \\
& \neq 0,
\end{aligned}
$$

contradicting the fact that $r_{d}\left(C_{t+1}(A)\right)=0$. We conclude that $B=0_{n-t}$ 
and hence that $A$ is normal.

Proof of Theorem 3. Once again we may assume that

$$
A=\left[\begin{array}{lll}
\lambda_{1} & & * \\
& \ddots & \\
0 & \lambda_{n}
\end{array}\right],
$$

so that $C_{m}(A)$ is also upper triangular. Let $\alpha \in Q_{m, s}, \gamma \in Q_{m, n}$, and assume $\gamma>\alpha$, i.e., $\gamma$ follows $\alpha$ in the lexicographic ordering. Moreover suppose that $|\alpha \cap \gamma|=m-1$, i.e., $\operatorname{Im} \alpha$ and $\operatorname{Im} \gamma$ overlap in $m-1$ places. Then if $|s|^{2}+|t|^{2}=1, s e_{\alpha}^{\wedge}+t e_{r}^{\wedge} \in G_{m}$ and

$$
\begin{aligned}
\mid\left(C_{m}(A)\left(s e_{\alpha}^{\wedge}+t e_{\gamma}^{\wedge}\right), s e_{\alpha}^{\wedge}+\right. & \left.t e_{\gamma}^{\wedge}\right)|\leqq| \lambda_{1} \cdots \lambda_{m} \mid \\
\left|\left(C_{m}(A)\left(s e_{\alpha}^{\wedge}+t e_{\gamma}^{\wedge}\right), s e_{\alpha}^{\wedge}+t e_{\gamma}^{\wedge}\right)\right|= & |s|^{2} C_{m}(A)_{\alpha, \alpha}+s \bar{t} C_{m}(A)_{r, \alpha} \\
& +t \bar{s} C_{m}(A)_{\alpha, \gamma}+|t|^{2} C_{m}(A)_{r, \gamma} \\
= & |s|^{2} \lambda_{\alpha}+t \bar{s} p(\gamma)+|t|^{2} \lambda_{\gamma},
\end{aligned}
$$

where $p(\gamma)=C_{m}(A)_{\alpha, \gamma}$;

$$
\left|\left(C_{m}(A)\left(s e_{\alpha}^{\wedge}+t e_{\gamma}^{\wedge}\right), s e_{\alpha}^{\wedge}+t e_{\gamma}^{\wedge}\right)\right|=\left.\left|\lambda_{1}\right|^{m-1}|| s\right|^{2} \lambda_{i}+\frac{t \bar{s}}{c} p(\gamma)+|t|^{2} \lambda_{j} \mid
$$

where $\left|\lambda_{i}\right|=\left|\lambda_{1}\right|$ and $c \neq 0$. From (18) we have

$$
|| s\left|{ }^{2} \lambda_{i}+\frac{t \bar{s}}{c} p(\gamma)+\right| t\left|{ }^{2} \lambda_{j}\right| \leqq\left|\lambda_{1}\right| \text {. }
$$

Applying the Elliptical Range Theorem to the matrix

$$
\left[\begin{array}{cc}
\lambda_{i} & \frac{p(\gamma)}{c} \\
0 & \lambda_{j}
\end{array}\right]
$$

tells us that unless $p(\gamma)=0$ there exists an $s$ and $t,|s|^{2}+|t|^{2}=1$, for which ||$s\left|{ }^{2} \lambda_{i}+t \bar{s} / c p(\gamma)+\right| t\left|{ }^{2} \lambda_{j}\right|>\left|\lambda_{1}\right|$. Thus

$$
C_{m}(A)_{\alpha, \gamma}=0 \quad \text { if } \quad \alpha \in Q_{m, s}, \gamma>\alpha \text {, and }|\alpha \cap \gamma|=m-1 .
$$

The elements of row $\alpha$ of $C_{m}(A)$ are the Plücker coordinates of the decomposable tensor $A_{\alpha(1)} \wedge \cdots \wedge A_{\alpha(m)}$ and therefore satisfy the quadratic Plücker relations:

$$
p(\alpha) p(\gamma)=\sum_{t=1}^{m} p(\alpha[s, t: \gamma]) p(\gamma[t, s: \alpha]), \quad s=1, \cdots, m .
$$

For $\gamma>\alpha,|\alpha \cap \gamma|=m-1$, we have seen that $p(\gamma)=0$. Let $\gamma>\alpha,|\alpha \cap \gamma| \neq m-1$. Pick $s$ in (19) so that $\alpha(s) \notin \operatorname{Im} \gamma$. Then 
$|\alpha[s, t: \gamma] \cap \alpha|=m-1$ so that the first factor in each summand of (19) is zero. Since $p(\alpha)=\lambda_{\alpha(1)} \cdots \lambda_{\alpha(m)} \neq 0$ we have $p(\gamma)=0$, i.e.,

$$
\left(C_{m}(A)\right)_{\alpha, \gamma}=0, \alpha \in Q_{m, s}, \alpha \neq \gamma .
$$

From (20),

$$
A_{\alpha(1)} \wedge \cdots \wedge A_{\alpha(m)}=\lambda_{\alpha(1)} \cdots \lambda_{\alpha(m)} e_{\alpha(1)} \wedge \cdots \wedge e_{\alpha(m)},
$$

which in turn implies the equality of the subspaces spanned by the two sets of vectors, i.e.,

$$
\left\langle A_{\alpha(1)}, \cdots, A_{\alpha(m)}\right\rangle=\left\langle e_{\alpha(1)}, \cdots, e_{\alpha(m)}\right\rangle, \alpha \in Q_{m, s}
$$

$\left(\left\langle x_{1}, \cdots, x_{m}\right\rangle\right.$ means the linear span of $\left.x_{1}, \cdots, x_{m}\right)$. Since $\left.s\right\rangle m$, for each $i \in\{1, \cdots, s\}$ there exist sequences $\alpha_{1}, \cdots, \alpha_{m} \in Q_{m, s}$ such that $\{i\}=\bigcap_{j=1}^{m} \operatorname{Im} \alpha_{j}$. If $\alpha \in Q_{m, s}$ then each $\alpha(i) \in\{1, \cdots, s\}, i=1, \cdots, m$, so that there exist sequences $\alpha_{1}, \cdots, \alpha_{m}$ such that $\{\alpha(i)\}=\bigcap_{j=1}^{m} \operatorname{Im} \alpha_{j}$. Therefore,

$$
\begin{aligned}
A_{\alpha(i)} \in \bigcap_{j=1}^{m}\left\langle A_{\alpha_{j}(1)}, \cdots, A_{\alpha_{j}(m)}\right\rangle & =\bigcap_{j=1}^{m}\left\langle e_{\alpha_{j}(1)}, \cdots, e_{\alpha_{j}(m)}\right\rangle,(\text { from }(21)) \\
& =\left\langle e_{\alpha(i)}\right\rangle .
\end{aligned}
$$

Hence $A=T+B$ where

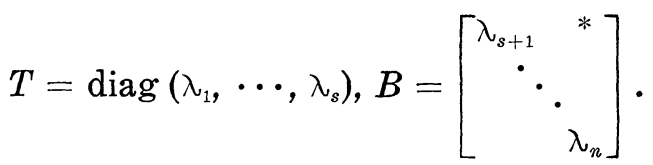

Finally, suppose there exists $u \in C^{n-s},\|u\|=1$, such that $|(B u, u)|>\left|\lambda_{1}\right|$. Let

$$
\begin{aligned}
& x_{i}=e_{i}, i=1, \cdots, m-1, \\
& x_{m}=0+u=\left(0, \cdots, 0, u_{1}, \cdots, u_{n-s}\right) .
\end{aligned}
$$

Then

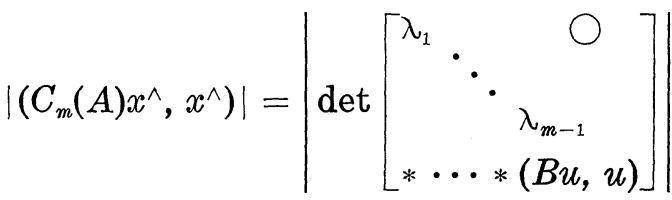

$$
\begin{aligned}
& =\left|\lambda_{1} \cdots \lambda_{m-1}(B u, u)\right| \\
& >\left|\lambda_{1} \cdots \lambda_{m}\right| \text {, }
\end{aligned}
$$

contradicting the hypothesis that $C_{m}(A)$ is decomposably spectral. Therefore $r(B) \leqq\left|\lambda_{1}\right|$ and by (12), $A$ is spectral.

To prove the converse, observe that $r_{d}\left(C_{m}(A)\right) \geqq\left|\lambda_{1}\right|^{m}$. Suppose 
$r_{d}\left(C_{m}(A)\right)>\left|\lambda_{1}\right|^{m}$. Then there exists $x^{\wedge} \in G_{m}$ such that

$$
\left.\mid C_{m}(A) x^{\wedge}, x^{\wedge}\right)\left.|>| \lambda_{1}\right|^{m} .
$$

Without loss of generality we can assume $x_{1}, \cdots, x_{m}$ are o.n. Let $U e_{i}=x_{i}, i=1, \cdots, U$ unitary, and compute that

$$
\begin{aligned}
\left|\left(C_{m}(A) x^{\wedge}, x^{\wedge}\right)\right| & =\mid\left(C_{m}\left(\left(U^{*} A U\right) e_{1} \wedge \cdots \wedge e_{m}, e_{1} \wedge \cdots \wedge e_{m}\right) \mid\right. \\
& =\left|\operatorname{det} U^{*} A U[1, \cdots, m \mid 1, \cdots, m]\right|
\end{aligned}
$$

Letting $B=U^{*} A U[1, \cdots, m \mid 1, \cdots, m]$, we have

$$
|\operatorname{det} B|>\left|\lambda_{1}\right|^{m} \text {, }
$$

so that $B$ has an eigenvalue $\tilde{\lambda}$ satisfying $|\tilde{\lambda}|>\left|\lambda_{1}\right|$. There exists a unitary $m$-square $V$ for which

$$
V^{*} B V=\left[\begin{array}{lll}
\tilde{\lambda} & & \\
& \ddots * & \\
& \ddots &
\end{array}\right] .
$$

Let $W=V+I_{n-m}$ and note that

$$
W^{*} U^{*} A U W=\left[\begin{array}{cc|c}
\tilde{\lambda} & & \\
& \ddots * & * \\
\bigcirc & * & \\
\hdashline * & *
\end{array}\right] .
$$

Let $X=U W ; X^{(1)}$, the first column of $X$, is a unit vector and

$$
\begin{aligned}
\left|\left(A X^{(1)}, X^{(1)}\right)\right| & =\left|\left(X^{*} A X\right)_{11}\right| \\
& =|\tilde{\lambda}|>\left|\lambda_{1}\right| .
\end{aligned}
$$

But this contradicts the fact that $r(A)=\left|\lambda_{1}\right|$. Therefore, $r_{d}\left(C_{m}(A)\right)=$ $\left|\lambda_{1}\right|^{m}$.

In the second part of Theorem 3 the hypothesis $s \geqq m$ is necessary. For, let

$$
A=\left[\begin{array}{lll}
1 & 0 & 0 \\
0 & 0 & 2 \\
0 & 0 & 0
\end{array}\right],
$$

and note that

$$
\frac{1}{2}\left(C_{2}(A)\left\{e_{1} \wedge e_{3}+e_{1} \wedge e_{2}\right\},\left\{e_{1} \wedge e_{3}+e_{1} \wedge e_{2}\right\}\right)=1>\lambda_{1} \lambda_{2}=0 .
$$


Also the hypothesis $s>m$ in the first part of Theorem 3 is necessary as the following examples illustrate:

$$
\begin{aligned}
A & =\left[\begin{array}{lll}
1 & 1 & 0 \\
0 & 1 & 0 \\
0 & 0 & 0
\end{array}\right], \\
C_{2}(A) & =\left[\begin{array}{lll}
1 & 0 & 0 \\
0 & 0 & 0 \\
0 & 0 & 0
\end{array}\right] ;
\end{aligned}
$$

then $r_{d}\left(C_{2}(A)\right)=1=\lambda_{1} \lambda_{2}$, but $r(A) \geqq r\left(\left[\begin{array}{ll}1 & 1 \\ 0 & 1\end{array}\right]\right)>1$;

$$
\begin{gathered}
A=\left[\begin{array}{lll}
1 & 1 & 1 \\
0 & \frac{1}{2} & 0 \\
0 & 0 & 0
\end{array}\right] \\
C_{2}(A)=\left[\begin{array}{lll}
\frac{1}{2} & 0 & 0 \\
0 & 0 & 0 \\
0 & 0 & 0
\end{array}\right] ;
\end{gathered}
$$

then $r_{d}\left(C_{2}(A)\right)=1 / 2=\lambda_{1} \lambda_{2}$, but $r(A) \geqq r\left(\left[\begin{array}{cc}1 & 1 \\ 0 & 1 / 2\end{array}\right]\right)>1$. Also observe that although Theorem 3 implies that if $C_{m}(A)$ is spectral, $m<s$, then $A$ is spectral, the converse is false. For example, let

$$
A=\left[\begin{array}{lll}
1 & 0 & 0 \\
0 & 1 & 0 \\
0 & 0 & 1
\end{array}\right]+\left[\begin{array}{ll}
0 & 2 \\
0 & 0
\end{array}\right]+\left[\begin{array}{ll}
0 & 2 \\
0 & 0
\end{array}\right] \text {. }
$$

Then $r(A)=1$ but $r\left(C_{2}(A)\right) \geqq r\left(\left[\begin{array}{ll}0 & 0 \\ 4 & 0\end{array}\right]\right)=2$ so that $C_{2}(A)$ is not spectral.

\section{REFERENCES}

1. I. C. Gohberg and M. G. Krein, Introduction to the Theory of Linaer Nonselfadjoint Operators, translations of Mathematical Monographs, Vol. 18, Amer. Math. Soc., 1969, p. 36 .

2. M. Goldberg, E. Tadmor and G. Zwas, The numerical radius and spectral metrices, Linear and Multilinear Algebra, 2 (1975), 209-214.

3. P. R. Halmos, A Hilbert Space Problem Book, Van Nostrand, 1967.

4. M. Marcus, Finite Dimensional Multilinear Algebra, Part II, Pure and Appl, Math. Series, Marcel Dekker, Inc., New York, 1975. 
5. H. Weyl, Inequalities between the two kinds of eigenvalues of a linear transformation, Proc. Nat. Acad. Sci., 35 (1949), 408-411.

Received August 12, 1976. Research of the second author was supported by a grant from the Air Force Office of Scientific Research, 72-2164.

UNIVERSITY OF ALASKA

AND

UnIVERSity of CALIForNia-SANTA Barbara 



\section{PACIFIC JOURNAL OF MATHEMATICS}

\section{EDITORS}

RICHARD ARENS (Managing Editor)

University of California

Los Angeles, California 90024

\section{J. DugunduI}

Department of Mathematics University of Southern California Los Angeles, California 90007

D. Gilbarg and J. Milgram

Stanford University

Stanford, California 94305

\author{
R. A. Beaumont \\ University of Washington \\ Seattle, Washington 98105
}

\section{ASSOCIATE EDITORS}
B. H. NeUmanN
F. WOLF
K. YosHIDA

\section{SUPPORTING INSTITUTIONS}

\author{
UNIVERSITY OF BRITISH COLUMBIA \\ CALIFORNIA INSTITUTE OF TECHNOLOGY \\ UNIVERSITY OF CALIFORNIA \\ MONTANA STATE UNIVERSITY \\ UNIVERSITY OF NEVADA \\ NEW MEXICO STATE UNIVERSITY \\ OREGON STATE UNIVERSITY \\ UNIVERSITY OF OREGON \\ OSAKA UNIVERSITY
}

\author{
UNIVERSITY OF SOUTHERN CALIFORNIA \\ STANFORD UNIVERSITY \\ UNIVERSITY OF HAWAII \\ UNIVERSITY OF TOKYO \\ UNIVERSITY OF UTAH \\ WASHINGTON STATE UNIVERSITY \\ UNIVERSITY OF WASHINGTON \\ AMERICAN MATHEMATICAL SOCIETY
}

The Supporting Institutions listed above contribute to the cost of publication of this Journal, but they are not owners or publishers and have no responsibility for its content or policies.

Mathematical papers intended for publication in the Pacific Journal of Mathematics should be in typed form or offset-reproduced, (not dittoed), double spaced with large margins. Please do not use built up fractions in the text of your manuscript. You may however, use them in the displayed equations. Underline Greek letters in red, German in green, and script in blue. The first paragraph or two must be capable of being used separately as a synopsis of the entire paper. Items of the bibliography should not be cited there unless absolutely necessary, in which case they must be identified by author and Journal, rather than by item number. Manuscripts, in triplicate, may be sent to any one of the editors. Please classify according to the scheme of Math. Reviews, Index to Vol. 39. All other communications should be addressed to the managing editor, or Elaine Barth, University of California, Los Angeles, California, 90024.

The Pacific Journal of Mathematics expects the author's institution to pay page charges, and reserves the right to delay publication for nonpayment of charges in case of financial emergency.

100 reprints are provided free for each article, only if page charges have been substantially paid. Additional copies may be obtained at cost in multiples of 50 .

The Pacific Journal of Mathematics is issued monthly as of January 1966. Regular subscription rate: $\$ 72.00$ a year (6 Vols., 12 issues). Special rate: $\$ 36.00$ a year to individual members of supporting institutions.

Subscriptions, orders for back numbers, and changes of address should be sent to Pacific Journal of Mathematics, 103 Highland Boulevard, Berkeley, California, 94708.

PUBLISHED BY PACIFIC JOURNAL OF MATHEMATICS, A NON-PROFIT CORPORATION

Printed at Kokusai Bunken Insatsusha (International Academic Printing Co., Ltd.), 8-8, 3-chome, Takadanobaba, Shinjuku-ku, Tokyo 160, Japan. 


\section{Pacific Journal of Mathematics}

Patricia Andresen and Marvin David Marcus, Weyl's inequality and

quadratic forms on the Grassmannian .......................

George Bachman and Alan Sultan, Regular lattice measures: mappings and

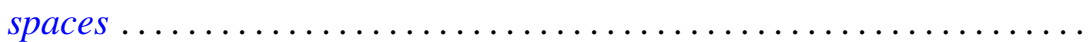

David Geoffrey Cantor, On certain algebraic integers and approximation by rational functions with integral coefficients ...................

James Richard Choike, On the value distribution of functions meromorphic in the unit disk with a spiral asymptotic value ..................

David Earl Dobbs, Divided rings and going-down................ 353

Mark Finkelstein and Robert James Whitley, Integrals of continuous

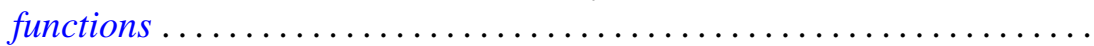

Ronald Owen Fulp and Joe Alton Marlin, Integrals of foliations on manifolds with a generalized symplectic structure ...............

Cheong Seng Hoo, Principal and induced fibrations .................

Wu-Chung Hsiang and Richard W. Sharpe, Parametrized surgery and

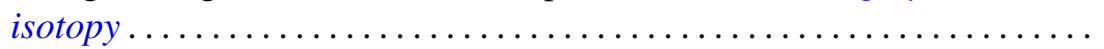

Surender Kumar Jain, Surjeet Singh and Robin Gregory Symonds, Rings whose proper cyclic modules are quasi-injective .................

Pushpa Juneja, On extreme points of the joint numerical range of commuting normal operators...

Athanassios G. Kartsatos, Nth order oscillations with middle terms of order $N-2$.

John Keith Luedeman, The generalized translational hull of a

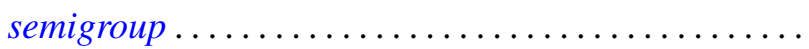

Louis Jackson Ratliff, Jr., The altitude formula and DVR's ...

Ralph Gordon Stanton, C. Sudler and Hugh C. Williams, An upper bound for the period of the simple continued fraction for $\sqrt{D}$...

David Westreich, Global analysis and periodic solutions of second order systems of nonlinear differential equations...

David Lee Armacost, Correction to: "Compactly cogenerated LCA

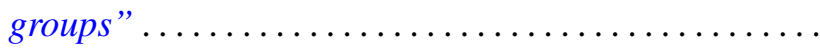

Jerry Malzan, Corrections to: "On groups with a single involution" .

David Westreich, Correction to: "Bifurcation of operator equations with unbounded linearized part" ...................... 